\title{
Creating an Intraoperative MRI Suite for the Musculoskeletal Tumor Center
}

\author{
Nathan W. Mesko MD, David M. Joyce MD, Hakan Ilaslan MD, \\ Michael J. Joyce MD
}

Received: 8 February 2015/Accepted: 10 June 2015/Published online: 17 July 2015

(C) The Association of Bone and Joint Surgeons \& 2015

\begin{abstract}
Background Altered anatomy in a previously irradiated surgical bed can make accurate localization of anatomic landmarks and local recurrence nearly impossible. The use of intraoperative MRI (iMRI) has been described in neurosurgical settings, but to our knowledge, no such description has been made regarding its utility for local recurrence localization in sarcoma surgery.

Case Description A 58-year-old female presented after previously undergoing two previous resection and reresection procedures of a myxoid liposarcoma located adjacent to her proximal femoral vasculature. After postoperative radiation therapy, she was referred to our

Each author certifies that he or she, or a member of his or her immediate family, has no funding or commercial associations (eg, consultancies, stock ownership, equity interest, patent/licensing arrangements, etc) that might pose a conflict of interest in connection with the submitted article.

All ICMJE Conflict of Interest Forms for authors and Clinical Orthopaedics and Related Research ${ }^{\circledR}$ editors and board members are on file with the publication and can be viewed on request.

Each author certifies that his or her institution approved the reporting of this case report, that all investigations were conducted in conformity with ethical principles of research, and that informed consent for participation in the study was obtained.

The study was performed at Cleveland Clinic, Department of Orthopaedic Surgery, Division of Musculoskeletal Oncology and Trauma, Cleveland, OH, USA.
\end{abstract}

N. W. Mesko ( $\varangle)$, D. M. Joyce, M. J. Joyce

Department of Orthopaedic Surgery, Cleveland Clinic, 9500

Euclid Ave. Crile Building, A-41, Cleveland, OH 44195, USA

e-mail: meskon@ccf.org

H. Ilaslan

Department of Diagnostic Radiology, Cleveland Clinic,

Cleveland, OH, USA institution where she underwent two additional reexcisions of local recurrences during a 3-year span, eventually undergoing a regional rotational muscle flap for coverage. Two years after her third reexcision procedure, she presented with two additional, nonpalpable surgical-bed local recurrences. After converting an MRI bed and scanner to allow for proximal thigh imaging in an iMRI surgical suite, the patient underwent a successful resection that achieved negative margins. To date, she remains without evidence of disease at 37 months.

Literature Review Real-time iMRI in neurosurgical studies has shown a high rate of residual disease leading to immediate subsequent reexcision, thus lending to improved rates of negative margin resection. To our knowledge, this is the first example using iMRI technology to remove a recurrent soft tissue sarcoma that otherwise was clinically nonlocalizable.

Clinical Relevance The use of an iMRI surgical suite can aid with identification of soft tissue nodules in conditions such as an altered tumor bed from prior resection and radiotherapy, which otherwise make recurrences difficult to localize. A team approach between administration, surgeons, and engineers is required to design and pragmatically implement the use of an MRI-compatible table extension to enhance existing iMRI surgical suite technology for extremity sarcoma resection procedures.

\section{Introduction}

One of the challenges for the musculoskeletal oncologist is accurate localization of masses deep to the fascia, a process that can be rendered more difficult by obesity, skewed anatomic landmarks, or abnormal tissue developing from previous irradiation or surgery. One solution proposed is the use of surgical navigation and CT-MRI fusion 
technology $[1,5-7,12,13]$, but this technique comes with inherent drawbacks. Although able to accurately locate a tumor based on landmarks, navigation does not allow for real-time confirmation of complete tumor excision. While samples of tumor bed tissue can be sent for real-time frozen section pathology review, and such analysis has shown high accuracy [3], difficulties have been described in distinguishing among scar tissue, healthy tissue, and tumor tissue in a surgical field previously altered by radiation or surgery [10].

The use of intraoperative MRI (iMRI) technology is a well-described neurosurgical technique that can enhance the probability of complete tumor excision, with several reports suggesting promising results for selected intracranial procedures $[4,8,11]$. Although these real-time MRI scans are characterized by small imaging fields, the visualized portal can be adequate for tumor localization and assessing adequacy of tumor resection in select orthopaedic oncology procedures where surrounding anatomy cannot be relied on to guide surgical resection, such as in the context of distorted anatomic fields through prior serial operations or radiation [9]. This may, in turn, allow the surgeon to more accurately confirm a negative margin resection with the use of real-time imaging, decreasing dependency on difficult to interpret frozen section analyses. To our knowledge, only one prior report of use of iMRI for soft tissue sarcoma has been published [9], which introduced this technique but did not discuss the technical and practical aspects surrounding planning and performing the resection.

We report our experience using a novel sarcoma resection technique using iMRI technology, and provide a step-by-step mechanism for converting a standard neurosurgery iMRI surgical suite into one that is pragmatic for excision of difficult to locate soft tissue sarcomas in an anatomically distorted field.

\section{Case Report}

A 58-year-old female had two margin-positive resections (the index resection in February 2006, and the first reexcision attempt in September 2007) at an outside musculoskeletal tumor center for a proximal and anterior thigh low-grade myxoid lipsarcoma, located adjacent to the femoral vasculature. After reexcision, she underwent $63 \mathrm{~Gy}$ adjuvant external beam radiation. In 2010, she presented to our institution with a second local recurrence, noted to have similar histologic features (Grade 2), and underwent a second reexcision in 2010; she then had a subsequent reexcision procedure 9 months later for a third recurrence outside the prior reexcision fields. After this, she underwent local soft tissue coverage using a rotational rectus abdominis flap. Two years later (in 2012), after the four previous operations and high-dose radiation, she had two additional MRI-documented recurrences in distinctly separate ipsilateral thigh locations, each measuring approximately $2 \mathrm{~cm}$ in diameter, and neither of which was palpable. The surgical field in which the local recurrence masses were found was characterized by substantial anatomic distortion, fibrosis, and radiation changes, with the femoral artery only localizable using Doppler ultrasound.

In preparation for a fifth attempt at local control, a neurosurgical iMRI (Siemens; Munich, Germany; 1.5 Tesla magnet on mobile track) was modified to perform an iMRIassisted sarcoma resection of the lower extremity recurrence areas. Partnering with hospital staff engineers, the operating table was modified, following blueprint designs, using MRI-compatible materials (ie, brass or plastic polymer screws and washers, plastic or carbon fiber platform) to accommodate patient positioning that would allow for adequate observation of the musculoskeletal field (proximal leg, pelvis) in the mobile iMRI scanner (Fig. 1). After immediate preoperative contrast MR images were obtained for comparison purposes, the patient was brought to the iMRI surgical suite and positioned on the modified iMRI table. Using primarily short Tau inversion recovery (STIR) axial sequencing cuts, the anterior tumor and medial tumor recurrence locations (Fig. 2) were confirmed before incision, by comparing immediate preoperative images with the same intraoperative STIR sequences. After identifying the tumor location following initial dissection, the surgical field was imaged a second time to determine an appropriate resection margin surrounding each recurrence (Fig. 3). After tumor excision, both specimens were removed from the surgical field, but kept in the iMRI scanning field (Fig. 4). A third scan then was done to confirm adequate resection of the field and to confirm the specimen with appropriate tissue margins. Through intraoperative frozen section assessment and postoperative permanent section review, the two specimens were confirmed histologically to have negative margins. No contrast was used for reasons described by Gould et al. [9], detailed in the Discussion. To date, the patient has no evidence of disease at 37 months after MRI-assisted resection.

\section{Discussion}

To our knowledge, there is only one other case series describing the use of iMRI for an orthopaedic tumor resection, although it lacks a descriptive modification process of the iMRI to make the surgical suite applicable for extremity surgery [9]. Our experience has shown that the application and successful use of a neurosurgical iMRI surgical suite in the field of musculoskeletal oncology can 


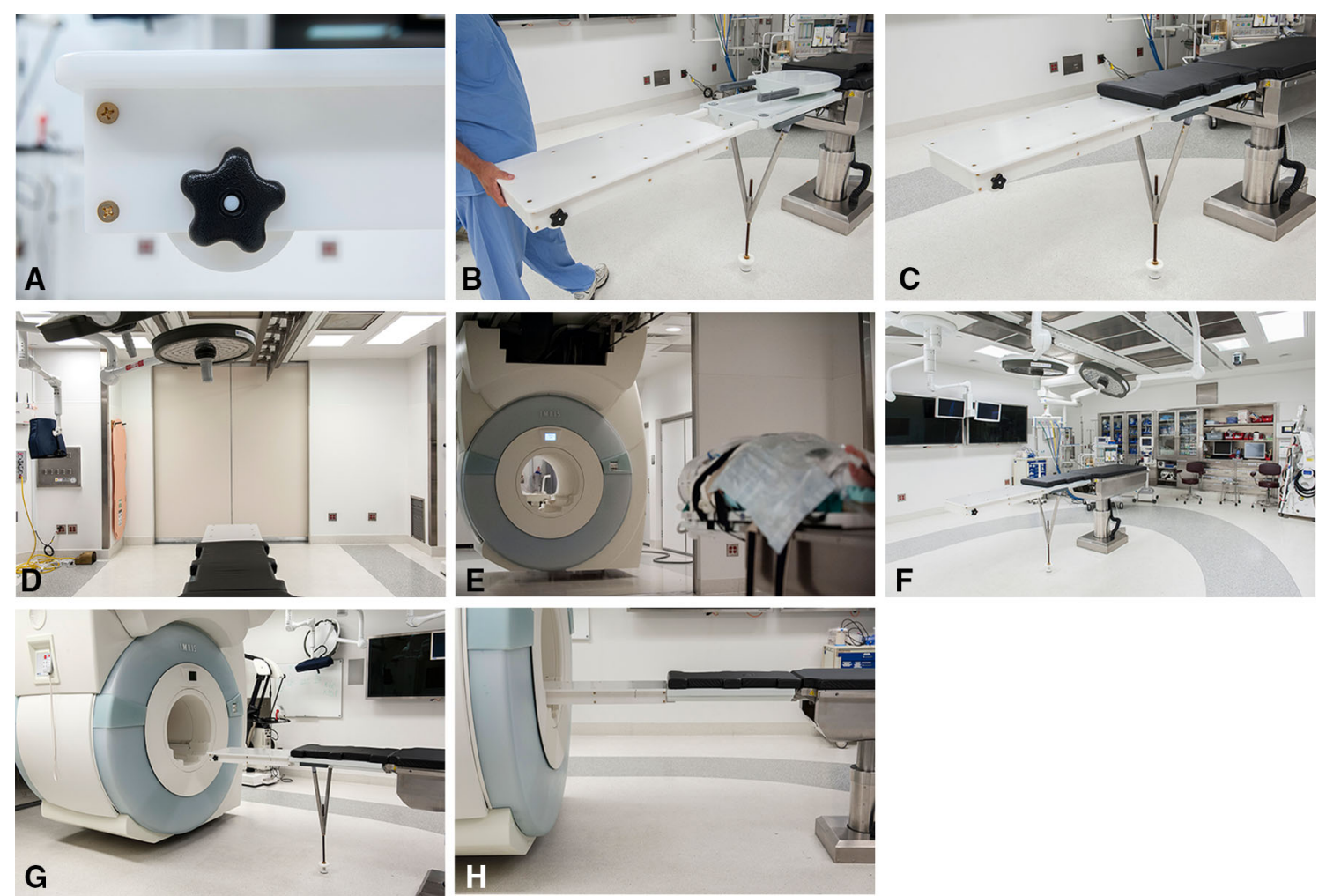

Fig. 1A-H In our modified operative suite, (A) the table extension (to accommodate the extremity) is made of nonmagnetic objects, such as brass, polymers, and plastics. This modification can be removed easily and does not effect the use for future neurosurgery cases. (B) A technician attaches the table modification that allows the patient's thigh or pelvis to enter the MRI scanner, with the patient in the supine position on the (C) modified table. (D) The MRI scanner is in a room adjacent to the operating room, and is moved to the operating room on (E) ceiling tracks thru a connecting door, with (F) all personnel, instruments, and equipment placed outside the gray peripheral line (magnetic field range) on the floor. An instrument count is performed before moving the instruments out of the magnetic field (any instrument unaccounted for is a potential projectile), while positive pressure flow prevents dirty airflow into the operating room suite while the magnet moves into position. (G) The scanner moves over the table extension, $(\mathbf{H})$ encircling the extension where the operative extremity is positioned. With each MRI scan the machine takes 15 minutes to move along the tracks (and a reciprocal 15 minutes to exit the room), with 10 to 15 minutes used to count instruments with each cycle. In this case, we performed three separate image acquisitions, taking approximately 45 minutes for each cycle, accounting for 135 minutes of operating room time. In addition, the image acquisition and interpretation took approximately 15 minutes each time. Use of MRI for resection guidance accounted for nearly 3 hours of additional operative time.

process, with the time commitment starting with initial design and modification phases to real-time implementation lasting up to 3 months. Furthermore, the estimated cost of table modification can range between USD 15,000 to USD 20,000. Once the appropriate patient selection criteria are met to determine the need for iMRI technology in extremity sarcoma excision surgery (Table 1), careful thought is required before implementing iMRI surgical suite modification (Fig. 5). The neurosurgical iMRI surgical suite (the designated context in which most institutions using iMRI technology have funded such capability) is largely designed for intracranial cases, with a focal imaging field centered over the brain. Because of this, special considerations are required for each unique surgical location, as operative table modifications may occur only in 

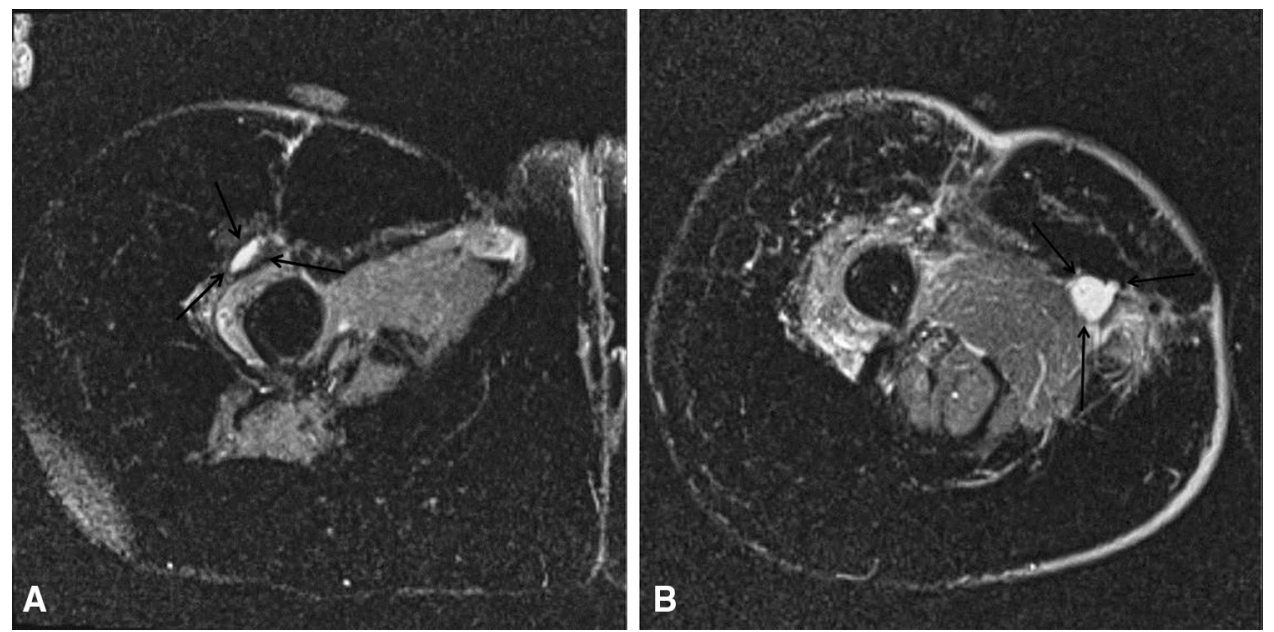

Fig. 2A-B Intraoperative axial STIR images through the right proximal thigh for localization show two small, hyperintense (A) anterior and (B) medial soft tissue masses (arrows) that are consistent with the known recurrent tumor. STIR $=$ short Tau inversion recovery.

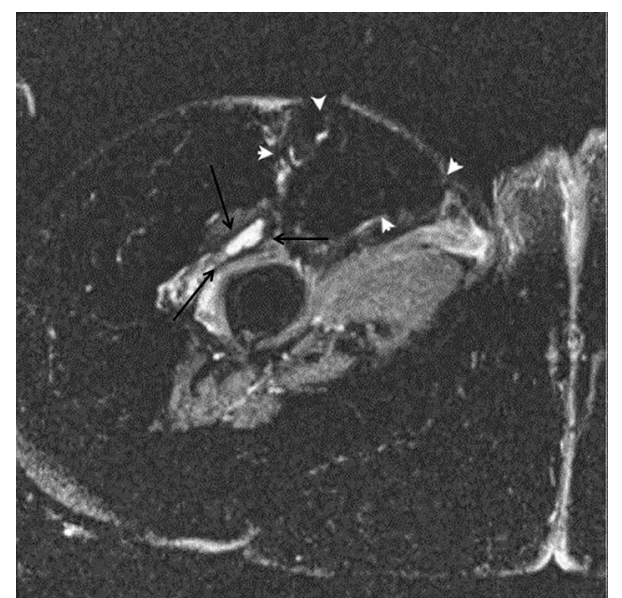

Fig. 3 An intraoperative axial STIR image through the right proximal thigh shows the surgical flap (arrowheads) elevated over the proximal recurrent soft tissue mass (arrows). STIR = short Tau inversion recovery.

line with the longitudinal axis, so as to accommodate the mobile iMRI machine tracking above the table (Fig. 1). In addition to the modification process, preoperative training, planning, and coordination dictates successful implementation of the iMRI surgical suite for specially modified purposes (Fig. 6). During the actual excision procedure, accuracy with intraoperative localization techniques is paramount to limit the total number of sequences needed. Vitamin E capsules are sealed in sterile plastic bags and must be changed frequently owing to their limited half-life. Sterility must be maintained through careful use of sterile sheets covering the extremity with each magnet entry in the room. Most important, efficiency is paramount during a case using iMRI, as lengthy (15-minute) image acquisition sequences and iMRI tracking over the table (30 minutes

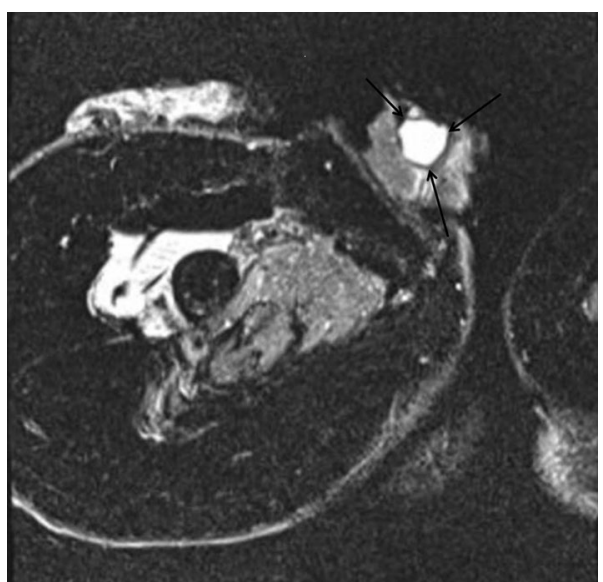

Fig. 4 A postoperative axial STIR image shows the resected tumor (arrows at top right) without residual mass in the surgical bed. STIR = short Tau inversion recovery.

with each acquisition) may create prolonged surgical times and the inherent potential downstream associations, such as infection, thromboembolic disease, or blood loss. The added time to the procedure, including image acquisition, image interpretation, and scanner mobilization, is in line with previous descriptions [4, 9].

In 2002, Gould et al. described the use of iMRI for resection of soft tissue sarcomas, although the three cases chosen to highlight involved skin-based dermatofibrosarcoma protuberans [9]. The reported benefits of iMRI were those of improved soft tissue resolution, lack of ionizing radiation, and multiplanar and postprocessing capabilities, all thought to be beneficial when trying to gain negative margins with resection of dermatofibrosarcoma protuberans, a notoriously difficult low-grade sarcoma on which to 
Table 1. Patient selection criteria for iMRI

\begin{tabular}{lc}
\hline Indications & Contraindications \\
\hline $\begin{array}{l}\text { Primary lesion(s) or recurrent lesion(s) is deemed to be resectable in a } \\
\text { manner that will not render a functionless extremity or compromise } \\
\text { margins to a degree that amputation is best offered }\end{array}$ & $\begin{array}{c}\text { Body habitus of the patient or the mass exceeds the limitations } \\
\text { of the iMRI table }\end{array}$ \\
$\begin{array}{l}\text { The lesion(s) is not readily palpable, and reliable identification using } \\
\text { landmark-based imaging measurements is impossible because of } \\
\text { radiation changes, postsurgical changes, or a combination thereof }\end{array}$ & $\begin{array}{c}\text { Previously implanted devices that are contraindicated in a } \\
\text { magnetic field (institution or MRI scanner-specific } \\
\text { protocols) }\end{array}$ \\
$\begin{array}{l}\text { Tumor location (in relation to essential structures required for a } \\
\text { functional limb) does not allow for conventional, unguided wide } \\
\text { resection, including normal surrounding tissue, to ensure excision } \\
\text { of the lesion }\end{array}$ & $\begin{array}{c}\text { Limited access to a musculoskeletal radiologist who is able to } \\
\text { aid witraoperative imaging interpretation }\end{array}$ \\
\hline
\end{tabular}
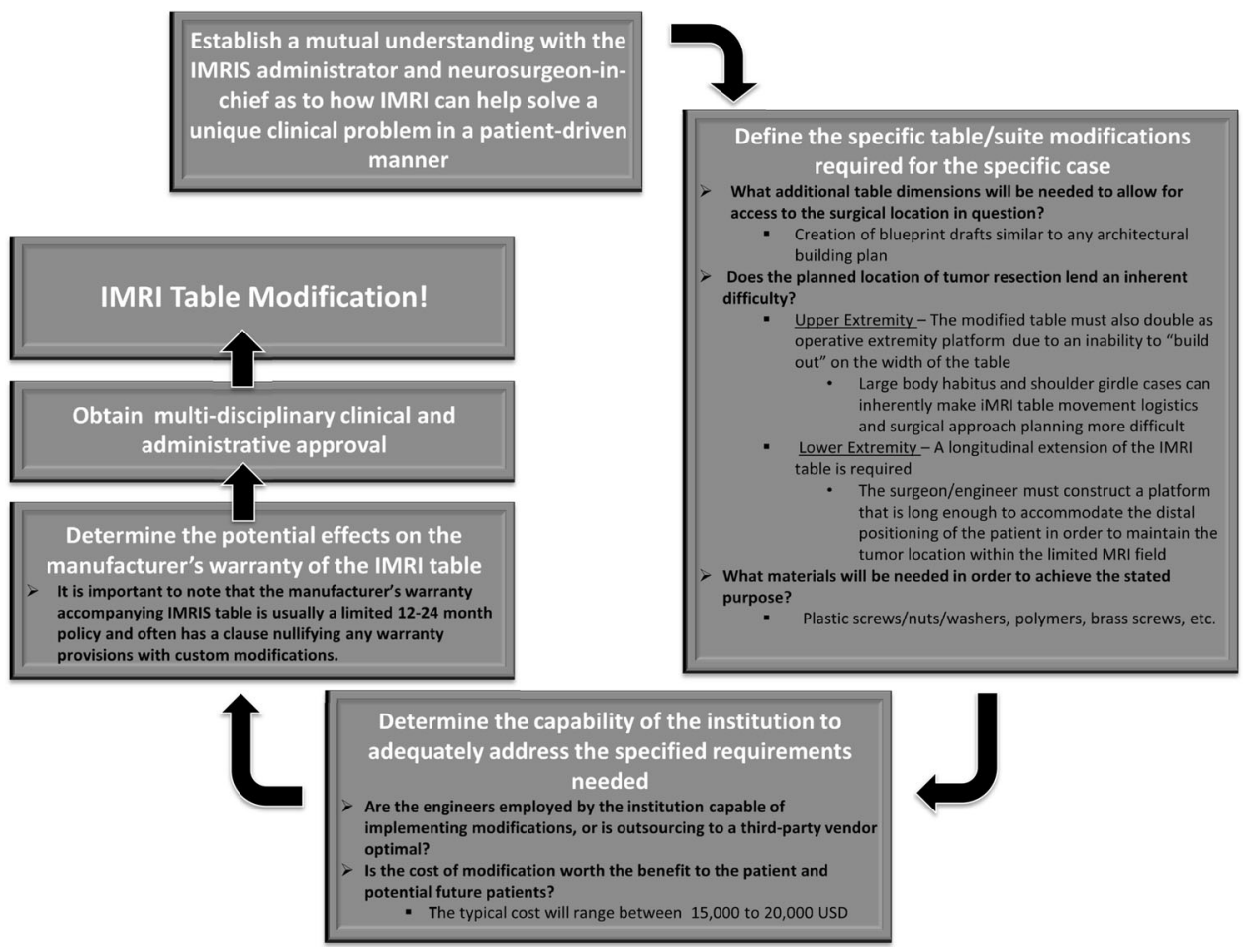

Fig. 5 Initiating the process of converting a neurosurgical iMRI table to being functionally acceptable for extremity tumor surgery requires multidisciplinary teamwork, financial backing, and discussion with the institutional engineers regarding the proper materials required to proceed with successful operating room suite modification. IMRI = intraoperative MRI; IMRIS = intraoperative MRI suite. gain adequate margins. No contrast was used, as specific axial STIR imaging sequences comparing preoperative MR images with intraoperative and preresection imaging were deemed adequate to determine the signal quality extent of the tumor lesion. Furthermore, concern that contrast washout may occur in a lengthy procedure, leaking into surrounding tissue, was thought to make it potentially more difficult to accurately confirm complete excision. Although this technique allowed distinct recognition of malignant tissue, no insight was provided regarding how to efficiently run an intraoperative MRI suite for soft tissue sarcoma resection. Similar to the methodology in the case series by
Gould et al. [9], our patient underwent iMRI assessment after resection to evaluate margins on the resected specimen and the remaining excision bed. A recent animal study [2] also showed efficacy of MRI technology in assessing resection margins, studying resection specimen margins in rats with intramuscular rhabdomyosarcoma. By comparing lesion margins defined on 1.5T MRI versus histopathology analysis, a high rate of agreement (Pearson coefficient 0.97 , average margin measured $0.3 \mathrm{~mm}$ in both analyses) between the two techniques was shown, thus lending indirect support to the utility of iMRI for use in the assessment of margins [2]. iMRI is better described in 


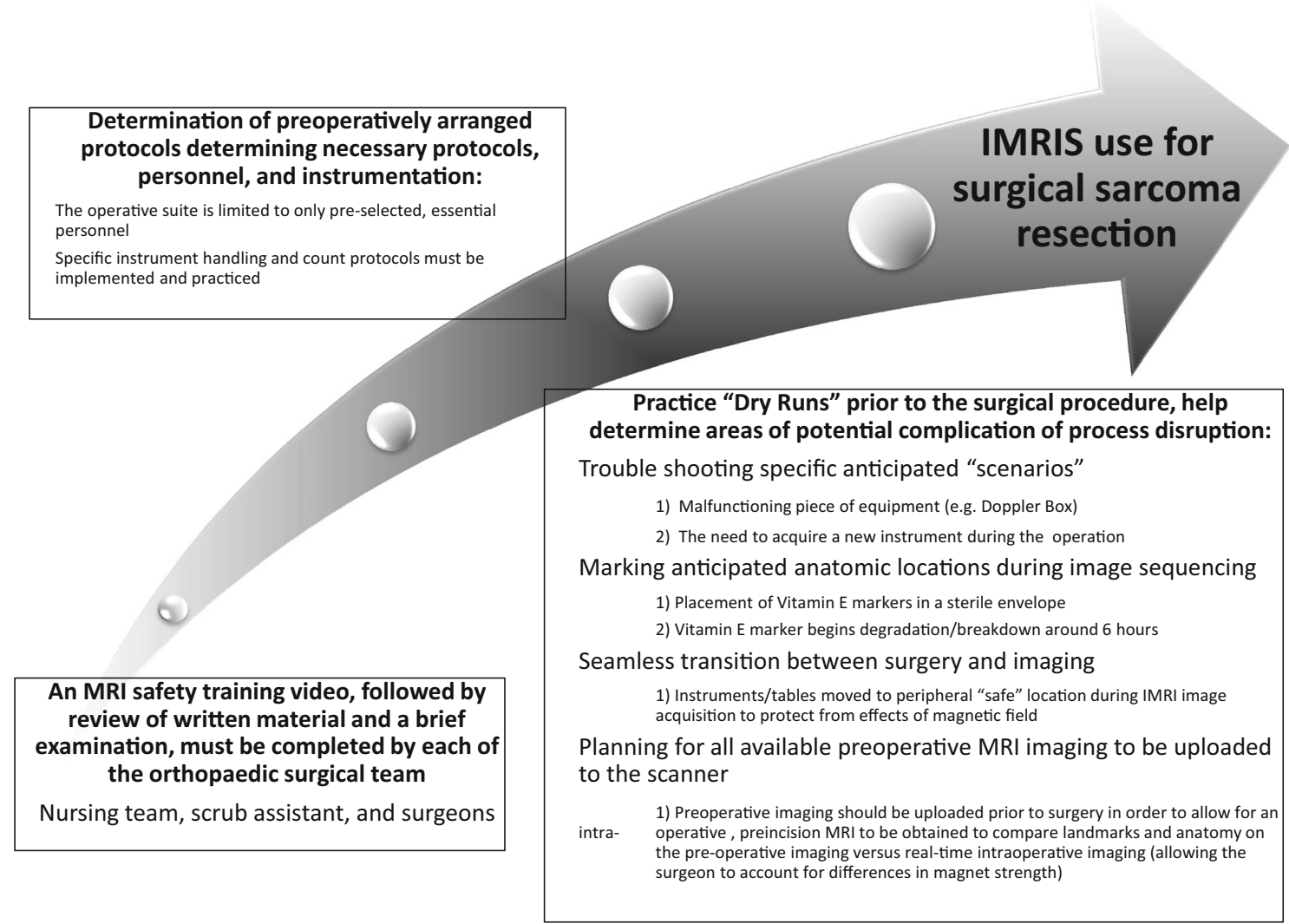

Fig. 6 After the iMRI surgical suite has been modified successfully, training and preparation for the real surgery are needed, anticipating for potential pitfalls or process adjustments intraoperative, and how to properly deal with these situations. Each essential participant is designated a specific role to help assure successful functioning of the iMRI surgical suite. IMRI = intraoperative MRI. neurosurgical settings for pituitary macroadenoma and temporal bone tumors, with suggested conclusions of improved surgical performance and a higher rate of wide margin resection $[8,12]$. In two separate small population prospective studies looking at 30 patients [4] and 73 patients [8] with pituitary macroadenomas, use of iMRI led to an increased extent of tumor resection and higher negative margin resection rate. In both studies, less than $2 / 3$ of patients $(59 \%$ and $66 \%$ ) had a negative margin resection denoted on the iMRI after the initial attempt at resection. Because of the high rate of residual tumor found on intraoperative imaging, the authors [4, 8] described repeat surgical resection as an effective way to decrease the residual tumor volume retained-an afforded benefit owing to real-time assessment with the patient under anesthesia. In contrast, a 2014 randomized control trial comparing standard neuronavigation versus iMRI for resection of glioblastoma showed no difference with respect to the extent of resection, clinical performance, or overall survival [11]. Despite this lack of observed difference, the authors maintained that iMRI is useful in the appropriate situation.

Given the drawbacks of substantial operating time (leading to higher rates of infection, blood loss, anesthesia time) and expense, the use of this technology for orthopaedic oncology should be limited. We believe this technology should be used only in situations where localization of the tumor through standard technique (referencing preoperative study measurements with local anatomic landmarks) is impossible because of fibrosis, prior muscle flaps, or other anatomically distorting features. If landmarks are palpable and the tumor can be clinically localized in a reliable fashion, then standard techniques are much more fiscally responsible and efficient. However, in situations such as with our patient, the intraoperative MRI suite concept can be a powerful instrument to aid the oncologic resection.

With multidisciplinary cooperation, creativity, and resourcefulness among the orthopaedic and neurosurgery surgical teams, the engineering department, and the institution's administration, a neurosurgical iMRI surgical suite can be modified to accomplish a musculoskeletal sarcoma excision requiring pinpoint localization. Use of iMRI offers a way to address a local soft tissue sarcoma recurrence in a previously irradiated field with anatomic distortion. Intraoperative MRI can benefit the surgeon and patient by helping to assure a negative margin resection without sacrificing vital structures. 
Acknowledgments We thank the Cleveland Clinic Engineering Department for help with the design and manufacturing of the intraoperative MRI suite operating table modification.

\section{References}

1. Aponte-Tinao L, Ritacco LE, Ayerza MA, Muscolo DL, Albergo JI, Farfalli GL. Does intraoperative navigation assistance improve bone tumor resection and allograft reconstruction results? Clin Orthop Relat Res. 2015;473:796-804.

2. Bellanova L, Schubert T, Cartiaux O, Lecouvet F, Galant C, Banse X, Docquier PL. MRI-based assessment of safe margins in tumor surgery. Sarcoma. 2014;2014:686790. doi: 10.1155/2014/ 686790. 2014 Feb 20. (Epub ahead of print]

3. Bhaker P, Mohan H, Handa U, Kumar S. Role of intraoperative pathology consultation in skeletal tumors and tumor-like lesions. Sarcoma. 2014:902104. doi: 10.1155/2014/902104. 2014 May 19. [Epub ahead of print]

4. Bohinski RJ, Warnick RE, Gaskill-Shipley MF, Zuccarello M, van Loveren HR, Kormos DW, Tew JM Jr. Intraoperative magnetic resonance imaging to determine the extent of resection of pituitary macroadenomas during transsphenoidal microsurgery. Neurosurgery. 2001;49:1133-1143; discussion 1143-1144.

5. Cheong D, Letson GD. Computer-assisted navigation and musculoskeletal sarcoma surgery. Cancer Control. 2011;18:171-176.
6. Cho HS, Oh JH, Han I, Kim HS. The outcomes of navigationassisted bone tumour surgery: minimum three-year follow-up. $J$ Bone Joint Surg Br. 2012;94:1414-1420.

7. Cho HS, Park IH, Jeon IH, Kim YG, Han I, Kim HS. Direct application of MR images to computer-assisted bone tumor surgery. J Orthop Sci. 2011;16:190-195.

8. Fomekong E, Duprez T, Docquier MA, Ntsambi G, Maiter D, Raftopoulos C. Intraoperative 3T MRI for pituitary macroadenoma resection: initial experience in 73 consecutive patients. Clin Neurol Neurosurg. 2014;126:143-149.

9. Gould SW, Agarwal T, Benoist S, Patel B, Gedroyc W, Darzi A. Resection of soft tissue sarcomas with intra-operative magnetic resonance guidance. J Magn Reson Imaging. 2002;15:114-119.

10. Hohenberger P, Schwarzbach MHM. Management of locally recurrent soft tissue sarcoma after prior surgery and radiation therapy. Recent Results Cancer Res. 2009;179:271-283.

11. Kubben PL, Scholtes F, Schijns OE, ter Laak-Poort MP, Teernstra OP, Kessels AG, van Overbeeke JJ, Martin DH, van Santbrink H. Intraoperative magnetic resonance imaging versus standard neuronavigation for the neurosurgical treatment of glioblastoma: a randomized controlled trial. Surg Neurol Int. 2014;5:70.

12. Nemec SF, Donat MA, Mehrain S, Friedrich K, Krestan C, Matula C, Imhof H, Czerny C. CT-MR image data fusion for computer assisted navigated neurosurgery of temporal bone tumors. Eur J Radiol. 2007;62:192-198.

13. Ritacco LE, Milano FE, Farfalli GL, Ayerza MA, Muscolo DL, Aponte-Tinao LA. Accuracy of 3-D planning and navigation in bone tumor resection. Orthopedics. 2013;36:e942-950. 